\title{
ENQUETTE SUR LA RÉPARTITION ET L'ÉVOLUTION DU PARASITISME A BUCEPHALUS POLYMORPHUS BAER, 1827 CHEZ LE MOLLUSQUE DREISSENA POLYMORPHA DANS LE SUD-EST DE LA FRANCE
}

\author{
Martine WALLET(1), A. LAMBERT (1)
}

(1) Laboratoire de Parasitologie comparée, Unité Associée au C.N.R.S. UA 698, Université des Sciences et Techniques du Languedoc, Place E. Bataillon, 34060 MONTPELLIER CEDEX - France Ce travail a bénéficié de l'aide financière du Conseil Supérieur de la Pêche /Convention CSP.USTL 82-316).

\section{RESUME}

Les premières données épidémiologiques sur la Bucéphalose larvaire à Bucephalus poly mcrphus dans le sud-est de la France sont présentées: elles concernent la répartition du mollus que vecteur Dreissena polymorpha le long de l'axe hydrographique faisant communiquer les bassins du Rhône et de la Garonne. L'évolution chez les mollusques des prévalences d'infestation et d'émission ont été suivies pendant quatre ans (1981-1984) dans une station témoin.

Distribution and evolution of parasitism of Bucepha/us polymorphus Baer, 1827 in the mollusc Dreissena polymorpha, first intermediate host, in south-east of France.

\section{SUMMARY}

The first epidemiological data of the larval infection with Bucephalus polymorphus in south-east of France are reported. The distribution of the mollusc Dreissena polymorpha, first intermediate host, in the water network between the Rhône and Garonne rivers, is studied. The evolution of parasitism in the mollusc was recorded for four years (1981-1984) in a reference station.

\section{INTRODUCTION}

La Bucéphaiose larvaire à Bucephalus polymorphus Baer, 1827, a fait en France l'objet de quelques travaux: dans le bassin de la Seine (de KINKELIN et Coll., 1966, 1967, 1968, 1969; TUFFERY, 1977) dans le nord-est (VOM SCHEIDT, 1984) et le sud-est (WALLET, 1984 ; WALLET et LAMBERT, 1984; WALLET et Coll., 1985)

Mais ces connaissances sont encore insuffisantes car elles ne permettent pas de cerner avec précision les conditions qui déterminent parfois, chez les Cyprinidae deuxièmes hôtes intermédiaires, une parasitose sévère avec des mortalités importantes.

Des éléments du cycle restent à étudier: ainsi chez le mollusque vecteur Dreissena polymorpha, les mécanismes de la sporocystogénèse et la cercariogénèse sont inconnus; ce lamellibranche représente encore une véritable "boîte noire" et l'influence des facteurs du milieu sur la production cercarienne reste donc à déterminer.

Par ailleurs, les interactions écologiques pouvant affecter les phénomènes de transmission sont mal connues dans le "compartiment cercaire".

II semble cependant, suite aux observations sur la biologie de la cercaire (WALLET, 1984; WALLET et Coll., 1985) que Dreissena polymorpha représente bien l'élément focalisateur de la parasitose. C'est pourquoi nous avons entamé nos recherches épidémiologiques en LanguedocRoussillon, à ce niveau du cycle

\section{MATERIEL ET MÉTHODES}

Le cadre géographique prospecté correspond à un axe qui fait communiquer par des canaux (navigables ou d'irrigation) le bassin inférieur du Rhône à celui de la Garonne (Figure 1): canal du Midi et canal du Bas-Rhône.

De part et d'autre de cet axe nous avons recherché le mollusque vecteur dans le réseau hydrographique naturel. 20 stations ont été prospectées. 
La station de Mauguio, située sur le canal d'irrigation du Bas-Rhône-Languedoc, près de Montpellier, a été suivie mensuellement pendant quatre ans (1981 à 1984).

Les échantillons prélevés pour déterminer la prévalence sont constitués d'une centaine d'individus minimum. Chaque mollusque est isolé au laboratoire dans un petit cristallisoir pour être contrôlé quotidiennement pendant 10 jours; on note ainsi les Dreissenes qui émettent effectivement des cercaires, ce qui permet de calculer la prévalence d'émission. La prévalence d'infestation correspondant au pourcentage d'individus parasités, qu'ils émettent ou non, est déterminée après dissection de tout l'échantillon.

A noter que la prévalence réelle est sous-estimée car il est pratiquement impossible de détecter les jeunes stades correspondant à une infestation récente.

\section{ASPECT BIOGEOGRAPHIQUE}

La figure 1 présente le cadre géographique prospecté.

Dreissena polymorpha n'a jamais été récoltée dans la partie occidentale de l'aire étudiée: le mollusque serait absent du canal du Midi dans la région de Castelnaudary et jusqu'à Toulouse. $D$. polymorpha n'a pas été observée dans le réseau hydrographique naturel adjacent à cet axe; c'est en particulier le cas de l'Orb, l'Hérault et l'Aude. Seul le Vidourle, dans sa partie aval (stations 18 et 19), semble faire exception à cette remarque. La Dreissene peut coloniser des milieux aménagés comme les lacs artificiels de retenue: ainsi le lac du Salagou (station 13) est très favorable à son développement.

Dans la majorité des stations où le mollusque est présent, le parasitisme à Bucephalus polymorphus existe, avec des prévalences parfois importantes comme le montre le tableau cidessous:

\begin{tabular}{|c|c|c|}
\hline STATIONS & $\mathrm{N}^{\circ}$ & PREVALENCES D'INFESTATION ET DATES \\
\hline $\begin{array}{l}\text { CANAL DU MIDI } \\
\text { PEZENS } \\
\text { VILLEDUBERT } \\
\text { MARSEILLETTE } \\
\text { LAREDORTE } \\
\text { ARGELIERS } \\
\text { LA CROISADE } \\
\text { CAPESTANG } \\
\text { BEZIERS } \\
\text { PORTIRAGNES } \\
\text { AGDE }\end{array}$ & $\begin{array}{r}1 \\
2 \\
3 \\
4 \\
5 \\
6 \\
7 \\
8 \\
9 \\
10\end{array}$ & 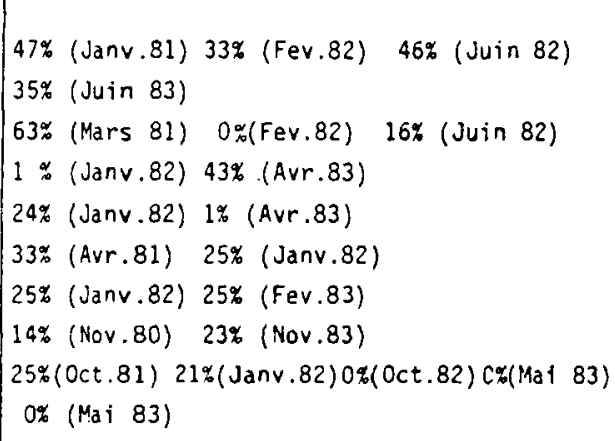 \\
\hline $\begin{array}{l}\text { CANAL DE JONCTION } \\
\text { ARGELIERS } \\
\text { SALLELES }\end{array}$ & $\begin{array}{l}11 \\
12\end{array}$ & $\begin{array}{l}3 \% \text { (Fẽv. 82) } \\
1 \% \text { (Fêv. 82) } 8 \% \text { (Fev. } 83 \text { ) }\end{array}$ \\
\hline$\frac{\text { LAC DU SALAGOU }}{\text { SALASC }}$ & $\begin{array}{l}13 a \\
13 b\end{array}$ & 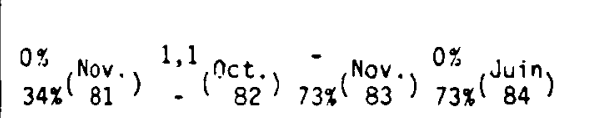 \\
\hline $\begin{array}{l}\text { CANAL DU RHONE A } \\
\text { SETE } \\
\text { FRANQUEVAUX } \\
\text { St. GILLES }\end{array}$ & $\begin{array}{l}14 \\
15\end{array}$ & $\begin{array}{rll}6 \% & \text { (Janv. 82) } & 4 \% \text { (Fév. } 83) \\
12 \% & \text { (Janv. 82) } & 8 \% \text { (Fév. } 83 \text { ) }\end{array}$ \\
\hline $\begin{array}{l}\text { CANAL DU BAS-RHONE } \\
\text { LUNEL-VIEL } \\
\text { LUNEL }\end{array}$ & $\begin{array}{l}16 \\
17\end{array}$ & $\begin{array}{l}2 \% \text { (Mars } 84) \\
0 \% \text { (Oct. 81) }\end{array}$ \\
\hline $\begin{array}{l}\text { VIDOURLE } \\
\text { VILIETELLE } \\
\text { LUNEL VIEL }\end{array}$ & $\begin{array}{l}18 \\
19\end{array}$ & 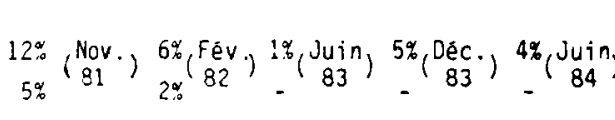 \\
\hline$\frac{\text { CONTRE CANAL DU }}{\frac{\text { RHONE }}{\text { BOLLENE }}}$ & 20 & $40 \%$ (Fev. 84 ) \\
\hline
\end{tabular}




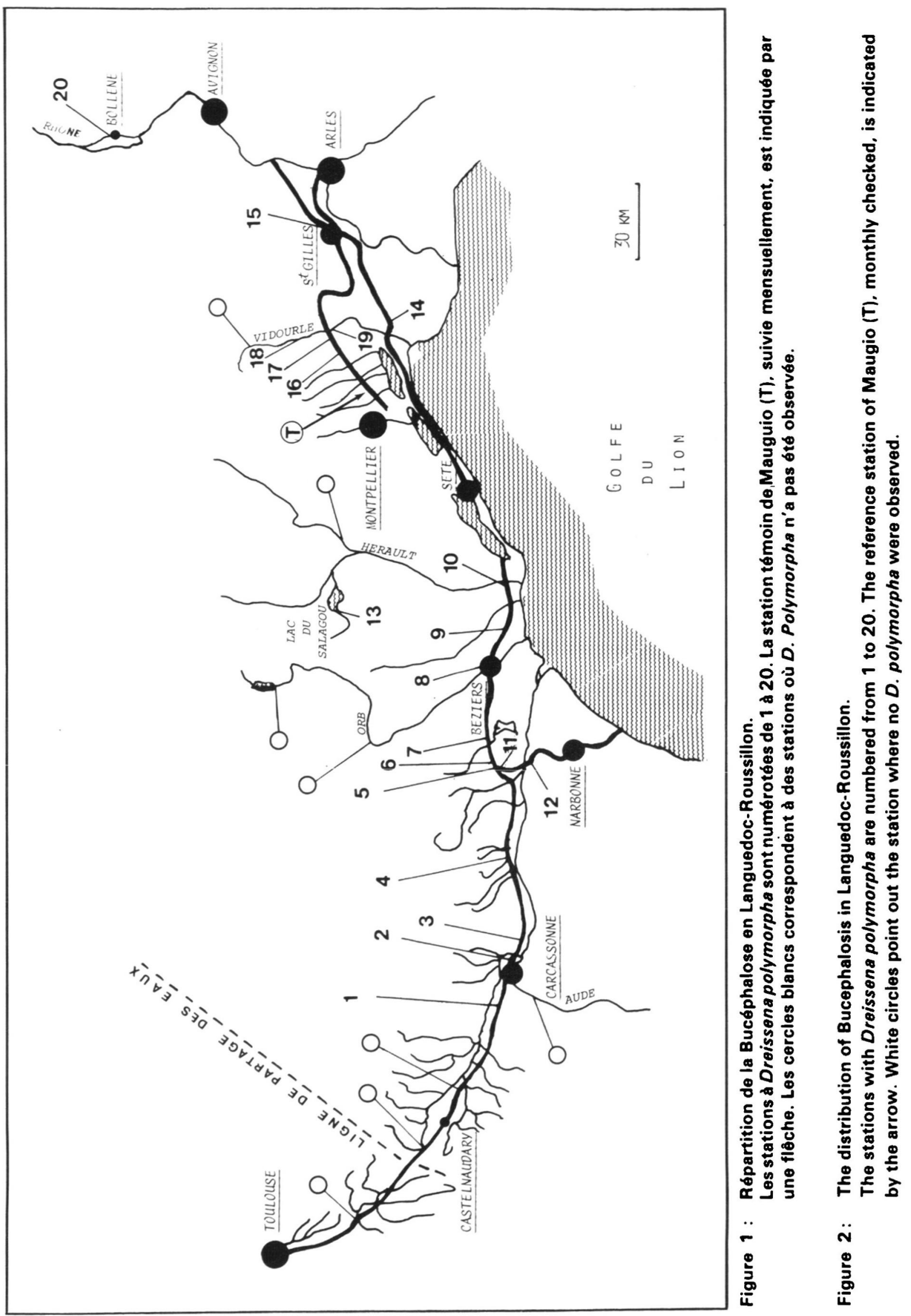




\section{ÉVOLUTION SAISONNIERE DU PARASITISME CHEZ DREISSENA POLYMORPHA}

La station témoin de Mauguio (station T, Fig. 1) a été suivie sur quatre années consécutives, de 1981 à 1984. L'évolution du parasitisme au niveau du mollusque est caractérisée par des fluctuations saisonnières (Fig. 2).

La prévalence d'infestation augmente brutalement pendant la période estivale; la prévalence des émissions suit une courbe paraltèle avec toutefois un décalage de 1 à 2 mois dans le temps. D'un point de vue épidémiologique c'est évidemment cette dernière notion qui est la plus importante: le même décalage existant dans le temps avec la courbe thermique, on peut supposer une relation de cause à effet entre l'élévation de la température de l'eau au printemps et les maxima d'émission de cercaires de l'été.

\section{CONCLUSION}

Ces observations concernant le parasitisme a Bucephalus polymorphus chez la Dreissene, dans le sud-est de la France, ne constituent qu'une première approche épidémiologique dont il faut retenir les éléments suivants

Dreissena polymorpha y colonise des milieux particuliers, en général aménagés (canaux de navigation ou d'irrigation et lacs de retenues). Elle paraît en revanche absente, à une exception près, des cours d'eau naturels.

Des travaux sur l'écologie et la biologie générale de ce mollusque existent (STANCZYKOWSKA 1964, 1977; MORTON, 1969-1971; MOUTHON, 1982); its restent cependant insuffisants pour expliquer, au niveau local, la répartition de la Dreissene qui, dans un même milieu apparent (le canal du Midi) semble absente à l'ouest de Castelnaudary. Seule une étude écologique locale permettrait de préciser les facteurs biotiques ou abiotiques limitants.

Les prévalences parasitaires à ce niveau du cycle sont parfois élevées par rapport à celles citées par de KINKELIN et Coll., 1969; TUFFERY, 1977 dans le Bassin parisien et par VOM SCHEIDT, 1984 dans le nord-est de la France. Mais, les différences observées ne sont pas significatives car, dans tous les cas, de fortes variations sont observées sur ces données, en fonction des stations et en fonction du temps: TUFFERY, 1977 signale sur le même site des prévalences qui évoluent de $28 \%$ en 1967 à $6,1 \%$ en 1976. Sur la Moselle VOM SCHEIDT (1984) donne 3 à $6 \%$ de moules parasitées au printemps et en été.

Cette variabilité du parasitisme semble être la règle générale: BATURO (1977) la retrouve en milieu lacustre, en Pologne $(0.9 \%$ en 1972 et $3,7 \%$ en 1973). Les prévalences rapportées dans notre enquête confirment cette variabilité.

Notre ignorance des modalités de la reproduction asexuée chez la moule (sporocystogénèse et cercariogénèse), et donc de l'influence des facteurs écologiques à ce niveau du cycle, empêche toute interprétation rationnelle. Nous ne savons pas plus dans quelle mesure les facteurs biotiques et abiotiques ont une action, directe, ou indirecte par l'hôte interposé, sur le développement du (ou des) sporocyste(s) et la maturation des cercaires.

Si de brusques variations peuvent être observées d'une année sur l'autre, il existe une fluctuation saisonnière nette des émissions cercariennes en relation avec les fluctuations thermiques de l'eau. Les maxima d'émissions se situent en été. Il est à ce sujet remarquable de noter la coincidence de ces maxima avec le fait que, à ce moment de l'année, les conditions photopériodiques sont celles qui expérimentalement provoquent un décalage du pic journalier d’émission vers la première heure d'éclairement (WALLET et Coll., 1985).

Ainsi se trouvent réunies, à cette période de l'année, des conditions pouvant favoriser des infestations massives de Cyprinidae. Mais ce ne sont certainement pas les seules. Les études sur la biologie de la cercaire (WALLET, 1984) ont montré que l'infestation des poissons est de type passif: son activité natatoire est faible, limitant sa dispersion propre qui se trouve ainsi plus influencée par les courants (courant exhalant du mollusque au moment de l'émission et courant du milieu environnant). Il s'avère donc difficile de cerner tous les facteurs qui déterminent les infestations massives des Cyprinidae car nos observations sur les émissions doivent être complétées par des travaux sur la production cercarienne au niveau des populations de mollusques.

Selon toute vraisemblance, il apparaît de plus en plus que les conditions amenant à une pathologie des poissons deuxième-hôte doivent être, en fonction des milieux, extrêmement variables: ces conditions devront être analysées, d'une part au niveau des Dreissena, à l'échelle des populations, et d'autre part au niveau des rapports pouvant exister entre ces populations et celles des Cyprinidae, en fonction de leur éthologie; ainsi aurons-nous peut-ètre un jour une explication précise au fait que les phénomènes pathologiques observés par de KINKELIN et al. (1967): TUFFERY (1977) ne se retrouvent pas, jusqu'à présent, dans le sud-est malgré des prévalences parasitaires parfois plus élevées au niveau du mollusque vecteur. 
3ull. Fr. Pêche Piscic. (1986) $300: 19-24-23-$

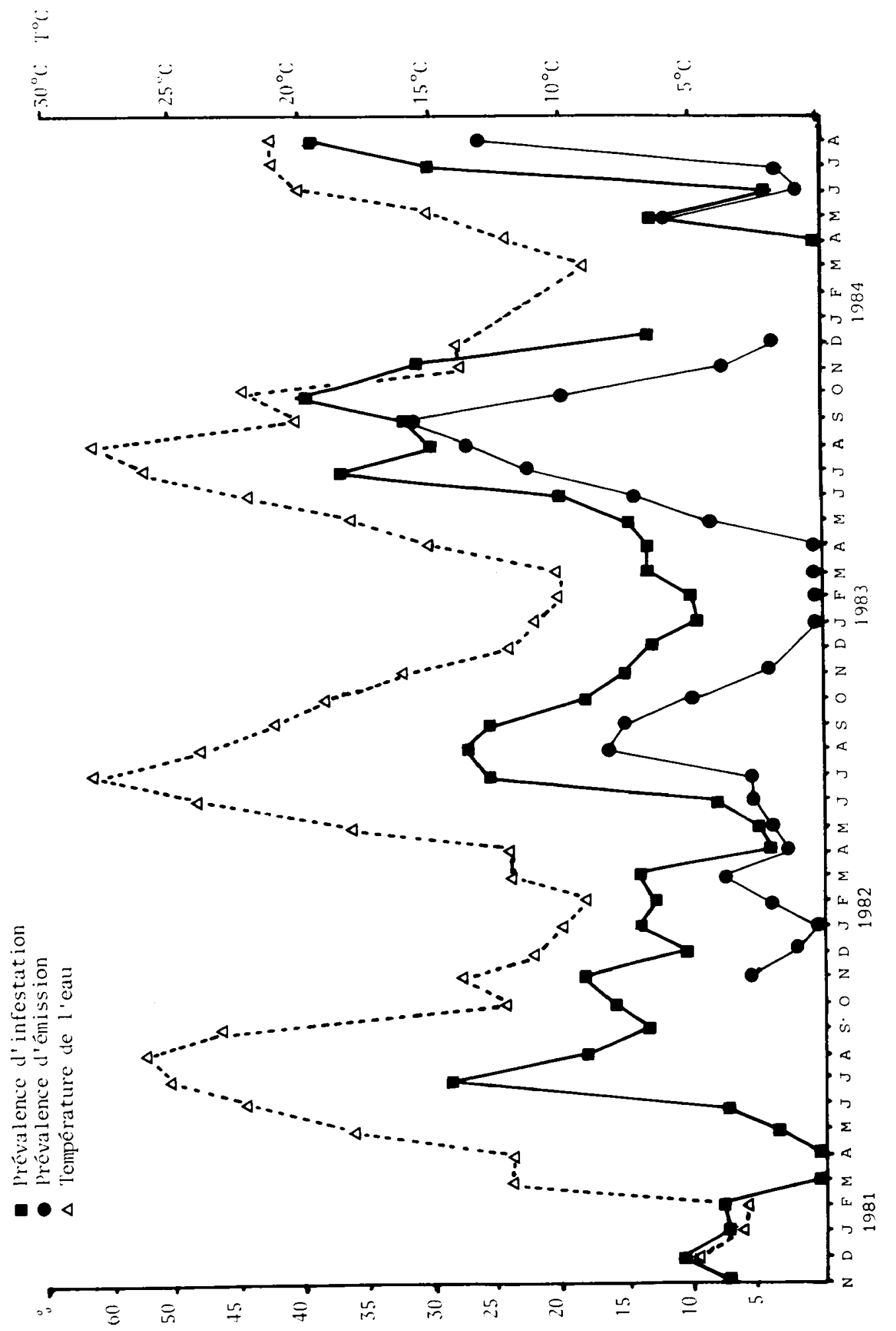

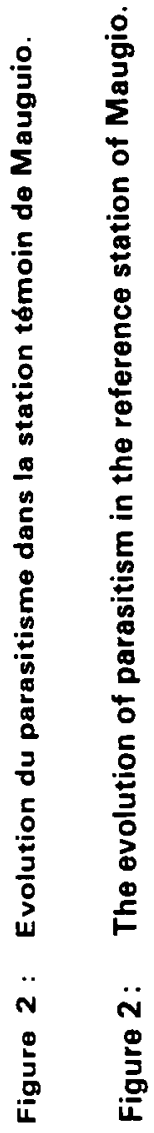


Le "compartiment cercaire" qui ne représente qu'un maillon de la chaîne épidémiologique de la Bucéphalose, nécessite encore, à lui seul, de nombreuses recherches.

Nous sommes donc encore loin de pouvoir prédire, à partir de quelques chiffres, les risques pathogènes. La nature hétéroxène de ce type de cycle complique les investigations qui doivent être menées selon deux directions principales:

- recherches fondamentales sur la biologie du parasite, sur les portions du cycle encore inconnues: biologie de l'adulte chez le Sandre (longévité, ponte...); étude du miracidium et modalités d'infestation de la Dreissene; sporocystogénèse, cercariogénèse chez ce mollusque, etc..

- recherches écologiques au niveau des populations pour comprendre l'évolution démographique du parasitisme, en relation avec la démographie et le comportement des hôtes.

Ces bases seront encore longues à acquérir, mais elles sont un préalable indispensable à toute tentative d'intégration et de modélisation.

\section{REMERCIEMENTS}

Les auteurs remercient $M$. Olivier DUCRET, Président du Club Halieutique. Président des A.A.P.P. de l'Hérault, pour les encouragements et l'aide qu'il n'a jamais cessé de nous apporter.

\section{BIBLIOGRAPHIE}

BATURO B., 1977. Bucephalus polymorphus Baer, 1827 and Rhipidocotyle illense (Ziegler 1883) (Trematoda, Bucephalidae): morphology and biology of developmental stages. Acta parasit. pol. 24 (20): 203-220.

KINKELIN P. de, BESSE P. et TUFFERY G., 1968. Une nouvelle affection nécrosante des téguments et des nageoires: la Bucéphalose larvaire à Bucephalus polymorphus (Baer, 1827). Bull. off. int. Epizoot., 69 : 1207-1230

KINKELIN P. de, BESSE P., JOLIVET G. et TUFFERY G., 1967. Rôle pathogène des cercaires de Bucephalus polymorphus Baer, 1827. Trématode Bucephalidae sur le peuplement piscicole du Bassin de la Seine. C.R. Acad. Sci. Paris, Ser. D., $264: 2321-2324$.

KINKELIN P. de, BESSE P., TUFFERY G. et JOLIVET G., 1966. L'épizootie du Bassin de la Seine-Yonne. La Pisciculture Francaise. 8: 18-24.

KINKELIN P. de, TUFFERY G., LEYNAUD G. et ARRIGNON J., 1969. La Bucéphalose larvaire à Bucephalus polymorphus (Baer, 1827): pathogénie, épizootiologie, possibilités d'intervention. Bull. Fr. Pisc., $234: 5-20$.

MORTON B.S., 1969. Studies on the biology of Dreissena polymorpha Pall. Proc. Malac. Soc. Lond., $38: 471-481$

MORTON B.S., 1971. Studies on the biology of Dreissena polymorpha Pall. V. Some aspects of the filter feeding and the effect of microorganisms upon the rate of filtration. Proc. Malac. Soc. Lond., 39 : 289-301.

MOUTHON J., 1982. Les mollusques dulcicoles. Données biologiques et écologiques. Clés de détermination des principaux genres de Bivalves et de Gastéropodes de France. Bull. Fr. Pisciculture, no spécial, mai 1982, 27 p.

STANCZYKOWSKA A., 1964. On the relationship between abundance aggregations and "conditions" of Dreissena polymorpha Pall. in 36 Mazurian lakes. Ekol. pol., Ser. A., 12: 653-690.

STANCZYKOWSKA A., 1977. Ecology of Dreissena polymorpha Pall. in lakes. Polskie Archwm Hydrobiol., $24: 461-530$.

TUFFERY G., 1977. Recherches sur la Bucéphalose à Bucephalus polymorphus Baer, 1827. Thèse de 3ème cycle, Université de Paris VI, 137 pp.

VOM SCHEIDT A., 1984. Etude de la Bucéphalose à Bucephalus polymorphus Baer, 1827 dans les rivières, fleuves et canaux du nord-est de la France. Thèse Doctorat Vétérinaire, E.N.V. d'Alfort, $111 \mathrm{p}$.

WALLET M., 1984. La Bucéphalose larvaire à Bucephalus polymorphus Baer, 1827 (Trematoda, Digenea). Biologie de la cercaire et aspects épidémiologiques dans le sud-est de la France. Thèse de 3ème cycle. Université des Sciences et Techniques du Languedoc. Montpellier. $188 p$.

WALLET M., LAMBERT A., 1984. Caractérisation de la cercaire de Bucephalus polymorphus Baer. 1827 (Trematode Bucephalidae). Chétotaxie et système excréteur. Ann. Parasit. Hum. Comp., 59: 583-588

WALLET M., THERON A., LAMBERT A., 1985. Rythme d'émission de cercaires de Bucephalus polymorphus Baer, 1827 (Trematoda, Bucephalidae) en relation avec l'activité de Dreissena polymorpha (Lamellibranche, Dreissenidae) premier hôte intermédiaire. Ann. Parasit. Hum. Comp. 60: 675-684. 